\title{
DIABETIC RETINOPATHY IN PATIENTS OF TYPE2 DIABETES MELLITUS WITH DIABETIC NEPHROPATHY
}

\section{Ophthalmology}

Dr. Debabrata Das MS Associate Professor, Deptt. OfOphthalmology, Midnapore Medical College, Paschim Medinipur, West Bengal, India.

\begin{tabular}{ll}
\hline Dr. Somedeb & MS Assistant Professor, Deptt. Of Ophthalmology, Midnapore Medical College, Paschim \\
Gupta* & Medinipur, West Bengal, India. *Corresponding Author \\
\hline
\end{tabular}

\begin{abstract}
To evaluate severity of diabetic retinopathy in patients of type 2 diabetes mellitus with diabetic nephropathy. This was a cross sectional study of 159 eyes of 80 patients aged above 40 years, diagnosed to have Type 2 DM with diabetic nephropathy. All the patients were investigated for albuminuria. The retinopathy was evaluated according to the Early Treatment Diabetes Retinopathy Study (ETDRS) classification into nonproliferative diabetic retinopathy (NPDR) and proliferative diabetic retinopathy (PDR). Diabetic macular edema was characterized as clinically significant macular edema (CSME) and non-CSME. The severity of retinopathy was correlated with nephropathy. In this study, mean age of the patients were $58.26 \pm 6.43$ years and male to female ratio 1.96:1. Out of 80 diabetic patients, $22(27.5 \%)$ patients had microalbuminuria and $58(72.5$ $\%)$ had macroalbuminuria. In this study, $67(83.7 \%)$ patients had diabetic retinopathy, out of which $14(20.9 \%)$ patients had mild NPDR, 20 (29.9\%) had moderate NPDR, 16 (23.9\%) had severe NPDR, 5(7.4\%) had very severe NPDR and 12 (17.9\%) had PDR. In 22 patients with microalbuminuria $6(27.3 \%)$ had mild NPDR, $4(5.0 \%)$ had moderate NPDR and none had maculopathy. In 58 patients with macroalbuminuria, 8 $(13.8 \%)$ had mild NPDR, $16(27.6 \%)$ had moderate NPDR, 16 (27.6\%) severe NPDR, 5 (8.6\%) has very severe NPDR, 4(6.9\%) had early PDR and $8(13.8 \%)$ had high-risk PDR, $14(24.1 \%)$ had CSME and $4(6.9 \%)$ had non-CSME. In our study, diabetic nephropathy patients with macroalbuminuria had more severe type of DR than patients with microalbuminuria.
\end{abstract}

\section{KEYWORDS}

Diabetic Retinopathy, Diabetic Nephropathy, Albuminuria

\section{INTRODUCTION}

India is stated to be the world's capital of diabetes mellitus (DM). The diabetic population in the country is estimated to be close to 69.9 million by 2025 and 80 million by 2030. Diabetes has become the fifth leading cause of blindness across the globe. It is estimated that about $70 \%$ of DM cases live in middle- and low-income countries. Diabetic retinopathy (DR) is one of the major causes for visual impairment and blindness among the diabetic patients. ${ }^{1} \mathrm{DR}$ is responsible for $4.8 \%$ of the 37 million cases of blindness throughout the world. ${ }^{2}$ In patients with DM, chronic hyperglycemia is the most important factor related to microvascular complications like retinopathy and nephropathy. ${ }^{3,4} \mathrm{As}$ globally the number of diabetics increases the development of microvascular complications also rises. Both diabetic retinopathy (DR) and nephropathy are typical microvascular complications of DM. Diabetic nephropathy (DN) is defined as a multifactorial pathological entity characterized by early renal hypertrophic changes to late stages of structural destruction of the glomeruli, renal vasculature, interstitium and tubules with clinical manifestations as overt continuous proteinuria, hypertension and declining glomerular filtration rates. As per a report of World Health Organization (WHO) the prevalence of nephropathy after 15 years of diabetes ranges between 17.7 and $56.6 \%$ in men and between 11.9 and $71 \%$ in women.. ${ }^{2}$ Persistent albuminuria is a predictor of development of clinical nephropathy. DN is the most frequent and dangerous complications of DM2, affecting about one-third of the patients. The common pathological abnormalities in the retinal and glomerular microvasculature are the progressive narrowing and eventual occlusion of vascular lumen within the retina and glomerulus. ${ }^{5,6}$ The DR and DN both have common risk factors, the duration of DM, poor glycemic control, dislipidemia and systolic hypertension. ${ }^{2}$ The damage caused by these microvascular complications of diabetes needs screening for both retinopathy and nephropathy. The detection of diabetic nephropathy is to estimate the excretion of microalbumin in $24 \mathrm{hrs}$ urine and for the detection of diabetic retinopathy (DR), fundus evaluation after pupillary dilatation. Many epidemiological studies suggest that patients with diabetic nephropathy are benefited from having regular ophthalmic evaluation. In this study, we evaluated severity of diabetic retinopathy in patients of type 2 diabetes mellitus with diabetic nephropathy

\section{MATERIALS AND METHODS}

This was a cross sectional observational study of 159 eyes of 80 patients diagnosed to have Type $2 \mathrm{DM}$ with diabetic nephropathy attending medicine OPD of a rural tertiary care hospital. The patients were referred to ophthalmology OPD for retinal evaluation and study period was from July 2017 to June 2018 . All the patients aged above 40 years having diabetic nephropathy and willing to participate in the study were included. The patients with hazy media, previous history of retinal laser therapy, pregnancy, retinal degeneration and dystrophies, uncontrolled hypertension, heart failure, urinary tract infection and obstruction, cystic kidney disease were excluded from the study. This research protocol was approved by the Institutional ethics committee. All the investigations were done according to Helsinki declaration. The full informed consent was taken from all participants.

General physical and systemic examinations were done in all the participants. Complete ophthalmological examination including visual acuity assessment and intraocular pressure was measured with Goldman tonometer. Slit lamp biomicroscopy and fundus examination were done. Fundus fluorescein angiography and optical coherence topography was done when indicated.

The DR was graded according to the ETDRS classification into mild, moderate, severe and very severe non-proliferative diabetic retinopathy (NPDR) and proliferative diabetic retinopathy (PDR) into early and high risk verities. Diabetic macular edema was characterized into clinically significant macular edema (CSME) and non-CSME.

All the patients were investigated by the physicians for diabetic nephropathy and were categorized as per albuminuria/24 hrs urine into microalbuminuria group $(30-300 \mathrm{mg} / 24 \mathrm{hr}$ urine $)$ and macroalbuminuria or proteinuria group $(>300 \mathrm{mg} / 24 \mathrm{hr}$ urine $)$.

Statistical analysis was done with software SPSS version 20.0 to analyze the data of the study. Differences were considered statistically significant at $\mathrm{p}<0.05$.

\section{RESULTS}

In this study, 159 eyes of 80 patients of diabetic nephropathy fulfilling inclusion criteria were evaluated for DR. The age of the patients were from 40 years to 76 years with mean age of $58.26 \pm 6.43$ years. There were 53(66.2\%) males and $27(33.8 \%)$ females with male to female ratio 1.96:1.The duration of diabetes was ranging from 2 to 18 years. Out of 80 diabetic patients, $16(20.0 \%)$ patients had diabetes for less than 5 years, $34(42.5 \%)$ patients for $5-10$ years and $30(37.5 \%)$ patients for more than 10 years.

Twenty-six (32.5\%) patient did not had any visual complaints and 54 $(67.5 \%)$ patients presented with dimness of vision. On funduscopic evaluation of 159 eyes for DR, microaneurysms were present in 133 
(83.6\%) eyes superficial retinal hemorrhages in $92(57.9 \%)$ eyes, soft exudates in $47(29.6 \%)$ eyes, hard exudates in $56(35.2 \%)$ eyes, deep hemorrhages in $51(32.0 \%)$ eyes, intraretinal microvascular abnormalities in 33(20.8\%) eyes venous beading in 49 (30.8\%) eyes, new vessels on the disc in $8(5.0 \%)$ eyes and new vessels elsewhere in $12(7.5 \%)$ eyes.

In our study, 67 (83.7\%) patients had diabetic retinopathy, out of which $14(20.9 \%)$ patients had mild NPDR, $20(29.9 \%)$ had moderate NPDR, $16(23.9 \%)$ had severe NPDR, 5(7.4\%) had very severe NPDR and 12 (17.9\%) had PDR (Table-1).

Table-1: Demographics

\begin{tabular}{|l|l|l|}
\hline Variables & $\begin{array}{l}\text { Number of patients } \\
(\mathrm{n}=80)\end{array}$ \\
\hline Mean age in years & & $58.26 \pm 6.43$ \\
\hline \multirow{3}{*}{ Sex } & Male & $53(66.2 \%)$ \\
\cline { 2 - 3 } & Femal & $27(33.8 \%)$ \\
\hline \multirow{5}{*}{ Duration } & 5 years & $16(20.0 \%)$ \\
\cline { 2 - 3 } & $5-10$ years & $34(42.5 \%)$ \\
\cline { 2 - 3 } & 10 years & $30(37.5 \%)$ \\
\hline \multirow{5}{*}{ Retinopathy } & Mild NPDR & $14(20.9 \%)$ \\
\cline { 2 - 3 } & Moderate NPDR & $20(29.9 \%)$ \\
\cline { 2 - 3 } & Severe NPDR & $16(23.9 \%)$ \\
\cline { 2 - 3 } & Very severe NPDR & $05(7.4 \%)$ \\
\cline { 2 - 3 } & PDR & $12(17.9 \%)$ \\
\hline Nephropathy & Microalbuminuria & 22 \\
\cline { 2 - 3 } & Macroalbuminuria & 58 \\
\hline
\end{tabular}

In this study 22(27.5\%) had microalbuminuria and $58(72.5 \%)$ had macroalbuminuria. In 22 patients with microalbuminuria $12(54.5 \%)$ patients did not have any retinopathy, $6(27.3 \%)$ had mild NPDR and 4 $(18.2 \%)$ had moderate NPDR. In 58 patients with macroalbuminuria $1(1.7 \%)$ patient did not have any retinopathy, $8(13.8 \%)$ had mild NPDR, $16(27.6 \%)$ had moderate NPDR, 16(27.6\%) severe NPDR, $5(8.6 \%)$ has very severe NPDR, $4(6.9 \%)$ had early PDR and $8(13.8 \%)$ had high-risk PDR. In our study severity of DR increased significantly with the level of albuminuria $(\mathrm{p}=0.0001)$.

In our study, 14(17.5\%) patients had CSME and 4(5.0\%) had nonCSME. Out of 22 patients of microalbuminuria none had maculopathy. In 58 patients with macroalbuminuria, 14 (24.1\%) patient had CSME and $4(6.9 \%)$ patient had non-CSME. The p-value was < 0.0001(Table2).

Table-2: Types of diabetic retinopathy with types of albuminuria

\begin{tabular}{|l|l|l|l|}
\hline \multicolumn{2}{|c|}{ Retinopathy } & \multicolumn{2}{c|}{ Nephropathy } \\
\cline { 3 - 4 } \multicolumn{2}{|c|}{$\begin{array}{l}\text { Microalbuminuria } \\
(\mathrm{n}=22)\end{array}$} & $\begin{array}{l}\text { Macroalbuminuria( } \\
\mathrm{n}=58)\end{array}$ \\
\hline \multirow{4}{*}{ NPDR } & Mild NPDR & 6 & 8 \\
\cline { 2 - 4 } & Moderate NPDR & 4 & 16 \\
\cline { 2 - 4 } & Severe NPDR & 0 & 16 \\
\cline { 2 - 4 } & Very severe NPDR & 0 & 5 \\
\hline PDR & Early PDR & 0 & 8 \\
\cline { 2 - 4 } & High-risk PDR & 0 & 4 \\
\hline
\end{tabular}

\section{DISCUSSION}

On evaluation of 80 patients in our study, $67(83.7 \%)$ patients had diabetic retinopathy, out of which 14 (20.9\%) patients had mild NPDR, $20(29.9 \%)$ had moderate NPDR, $16(23.9 \%)$ had severe NPDR, $5(7.4 \%)$ had very severe NPDR and $12(17.9 \%)$ had PDR We found that most of the diabetic patients having nephropathy also had retinopathy either in nonprolifrative $(68.7 \%)$ or in proliferative stage $(15.0 \%)$ and only few had normal fundi $(16.3 \%)$. The same findings were reported in the Trial to Reduce Cardiovascular Events with Aranesp Therapy (TREAT) study ${ }^{8}$, which stated DR was common in diabetic patients having nephropathy. Lee at al ${ }^{10}$ also showed a direct association between DR and DN.

In this study 22(27.5\%) had microalbuminuria and $58(72.5 \%)$ had microalbuminuria or proteinuria. In 22 patients with microalb uminuria, $6(27.3 \%)$ had mild NPDR and $4(5.0 \%)$ had moderate NPDR. In 58 patients with macroalbuminuria, $8(13.8 \%)$ had mild NPDR, $16(27.6 \%)$ had moderate NPDR,16(27.6\%) severe NPDR,5(8.6\%) has very severe NPDR, $4(6.9 \%)$ had early PDR and
$8(13.8 \%)$ had high-risk PDR. Therefore in our study severity of DR increased with the level of albuminuria. The prevalence of proliferative retinopathy was significantly higher in patients with macroalbuminuria as compared to those with microproteinuria. Singh SK et al ${ }^{11}$ showed that increase in urinary albumin excretion correlates with the development of proliferative retinopathy. In our patients with type 2 diabetes nephropathy, more patients had NPDR than PDR and less number of patients has normal fundi corroborating with the study of Bello et al

Out of 22 patients of microalbuminuria none had maculopathy. In 58 patients with macroalbuminuria,14 (24.1\%) patients had CSME and 4 (6.9\%) patients had non-CSME.

\section{CONCLUSION}

Hence, it is obvious that diabetic patient having nephropathy have increase likelihood that these patients may have diabetic retinopathy. Therefore, diabetic patients presenting to a physician should have to be referred to an ophthalmologist for a funduscopic evaluation of any retinopathy present or not.

\section{REFERENCES}

1. Pandey SK, Sharma V. World diabetes day 2018: Battling the Emerging Epidemic of Diabetic Retinopathy. Indian J Ophthalmol 2018;66:1652-3

2. Wirta O, Pasternack A, Mustonen J, Laippala P, Lähde Y: Retinopathy isindependently related to microalbuminuria in type 2 diabetes mellitus. Clin Nephrol 1999, 51:329-33

Unnikrishnan RI, Rema M, Pradeepa R, Deepa M, Shanthirani CS, Deepa R,Mohan V: Prevalence and risk factors of diabetic nephropathy in an urban South Indian population: the Chennai Urban Rural Epidemiology Study (CURES 45). Diabetes Care 2007 30:2019-2024.

4. Prevalence of small vessel and large vessel disease in diabetic patients from 14 centers. The World Health Organization Multinational Study of Vascular Disease in Diabetics. Diabetes Drafting Group. Diabetologia 1985, 28:615-640.

5. Sobngwi E, Mbanya JC, Moukouri EN, Ngu KB: Microalbuminuria and retinopathy in diabetic population of Cameroon. Diabetes Res Clin Pract 1999, 44:191-196

6. Kaiser, N.; Sasson, S.; Feener, E.P.; Boukobza-Vardi, N.; Higashi, S.; Moller, D.E. Davidheiser, S.;Przybylski, R.J.; King, G.L. Differential regulation of glucose transport and transporters by glucose in vascular endothelial and smooth muscle cells. Diabetes 1993, 42, 80-89.

7. Mizutani, M.; Kern, T.S.; Lorenzi, M. Accelerated death of retinal microvascular cells in human and experimental diabetic retinopathy. J. Clin Investig. 1996, 97, 2883-2890.

8. Cavalieri GC, Lima MC, Nazario NO, Lima GC. Risk factors for diabetic retinopathy: case-control study. Int J Retina Vitreous, 2016;2:2

9. Bello NA, Pfeffer MA, Skali H, McGill JB, Rossert J, Olson KA et al. .Retinopathy an clinical outcomes in patients with type 2 diabetes mellitus, chronic kidney disease, and clinical outcomes in patents with type 2 diabetes mellitus, chr

10. Lee WJ, Sobrin L, Kang MH, Seong M, Kim YJ, Yi JH, Miller JW, Cho HY. Ischemic diabetic retinopathy as a possible prognostic factor for chronic kidney disease progression. Eye $2014 ; 28(9): 1119-25$.

11. Singh SK,Bhere A,Singh MK.Diabetic retinopathy and microalbuminuria in lean type 2 diabetes mellitus.J Asso Physicians India.2001;49:439-4 